\title{
Cooperative Game Theory Methods in the Analysis of Economic and Political Interaction at the International Level
}

\author{
Pavel V. Konyukhovskiy ${ }^{1}$ and Victoria V. Holodkova ${ }^{2}$ \\ 1 Herzen State Pedagogical University of Russia, \\ Institute of Economics and Management, \\ 48 Moika Embankment, St.Petersburg, 191186, Russia \\ E-mail: aura4004@gmail.com \\ $W W W$ home page: \\ https://www.herzen.spb.ru/main/structure/inst/iem/1413967378/1419584026/ \\ 2 Saint-Petersburg State University, \\ Faculty of Economic, Department of Cybernetic Economic \\ 13 B Universitetskaya Emb., St.Petersburg 199034, Russia \\ E-mail: holodkova_v@mail.ru
}

\begin{abstract}
The main attention is focused on the application of the methods of the theory of cooperative games to the analysis of the relationship between the leading actors in international politics, or, as they say, the centers of power. One of the specific features of the modern world is the "triple type" of conflicts. Namely, at different levels of relationships, conflict situations with three participants (players) are often observed. Such situations are objectively characterized by the formation of possible paired coalitions, rejecting the third. The main idea of the proposed approach is the transition from cooperative games with deterministic values of characteristic functions to their counterparts with stochastic values. One of the possible concepts of solutions for stochastic cooperative games is associated with the extension of the ideas of the bargaining set to them. Problems of development and interpretation of this concept in the case of a triple conflict of international centers of power. An essential advantage of this approach is the possibility of meaningful interpretations of the significance level, at which the conditions for the rationality of the shares of the players should be ensured, taking into account the non-determinism of their utilities given by the characteristic function.
\end{abstract}

Keywords: game theory, cooperative games, stochastic cooperative games, bargaining set, intercountry interaction, centers of power.

\section{General set of the problem}

This paper is a development of the research begun in the works of the authors, Konyukhovskiy, Holodkova (2017).

The situation of relations in the US-China-Russia triangle claims to be the "brightest and most obvious" example. However, some other situations are also relevant to the "triple configuration". In particular: USA - Russia - Western Europe, Russia - Turkey - Iran, etc.

The political atmosphere of the early $20^{s}$ of the $21^{\text {st }}$ century can be briefly described with the phrase "general expectation of global changes in the world order". There are many works, both scientific and publicistic, trying to give a theoretical substantiation of the current situation. However, most of them are of an abstract, speculative nature and do not rely on any serious quantitative mathematical models.

https://doi.org/10.21638/11701/spbu31.2021.15 
At the moment, a certain generally accepted (consensus) set of theses has been formed regarding the relationship between economics and politics. On the one hand, it is recognized that objective economic processes underlie fundamental political phenomena. At the same time, few people question the thesis that political processes radically affect the state of both the macroeconomic systems of individual countries and regions, and the world economic system as a whole.

The purpose of the research, the results of which are reflected in this paper, is to develop methods for analyzing the patterns of interaction between world centers of power, which make it possible to obtain realistic adequate explanations of the causes of conflicts and confrontation, on the one hand, and possible points of reaching mutual agreements, on the other.

\section{Previous research}

It is necessary to note three groups of scientific sources on which this study is based.

First, there are works that consider cooperative games with transferable utility and concepts for their solution. Secondly, these are papers and monographs devoted to the problems of the application of cooperative games in the field of political science, political-economic and socio-political problems. Finally, these are studies devoted to the problems of the main subject area of current research. These, as already mentioned, include the patterns of interaction at the international level of countries and interstate coalitions. The principles of forming such coalitions, the logic of their cooperation and confrontation.

The theoretical and mathematical apparatus of the paper is based on the fundamental works of Shapley, Shubik (1954), Banzhaf (1965), and others.

This work is based on two fundamental theoretical ideas. First, the application of the toolkit of stochastic cooperative games to modeling political and economic systems and the relations of their subjects. Secondly, the implementation of the concept of a bargaining set for stochastic cooperative models.

Approaches to the definition of stochastic cooperative games and games and the analysis of their properties are devoted to the works Suijs, Born (1999), Suijs (1999), Suijs, Born (1999), Yeung, Petrosyan (2006), Konyukhovskiy (2012), Konyukhovskiy, Holodkova (2017)..

In terms of the concept of a bargaining set and the possibilities of its extension to stochastic cooperative games, this paper is based on the classic work of Aumann, Maschler (1961).

The scientific direction associated with the application of the methods of the theory of cooperative games to the modeling of political and political-economic research has a fairly rich history at the moment. An important place among the sources is occupied by the works of Penrose (1946), Johnston (1978), Deegan, Packel (1978), Holler, Packel (1983).

They reflect the problem of meaningful interpretation of game models in relation to the specifics of the behavior of political and socio-political systems in conditions of conflict of interest. Also, these works formulate "classical" approaches to assessing the influence of players and the coalition. For example, the calculation of the index of influence of various parliamentary coalitions. This is of fundamental importance for the subsequent development of methods for constructing the characteristic functions 
of games that describe the interaction of subjects of the system of international relations.

Among the Russian studies devoted to the application of the theory of cooperative games in models of political interaction, one should note the works of Aleskerov, Kravchenko, (2008). They are devoted to the analysis of the distribution of influence between factions in various compositions of the State Duma of the Russian Empire. The article by Sokolova (2008) is devoted to the calculation of influence indices and examines them on the examples of the Council of Ministers of the European Union and the modern Russian State Duma.

The current political, political, economic and socio-economic situation in the world is characterized by increased turbulence, dynamic changes in the roles and capabilities of traditional players, and the emergence of new players. Declarations of instability, along with calls for the restoration of stability, have become commonplace in international diplomatic discourse. Moreover, the very meanings invested in the term "stability" look like something fuzzy and vague. How to correctly interpret goals for achieving stability. Should we try to return to a pre-existing stable state, if possible, reduce the turbulence and variability of the current situation, or look for some "new stability" in the future?

This set of problems is reflected in modern scientific and applied research. In particular, it is worth paying attention to the following works.

The monograph Keersmaeker (2015) examines the problems of the concepts of "unipolar" and "multipolar" world. The author expresses doubts about their correctness and formulates a position suggesting a refusal to use the concept of "polarity". These questions are linked to the topic of this study, since it formulates versions of cooperative game-theoretic models that partially explain the mechanisms of the transition from a unipolar world to a multipolar one.

The monograph Degterev (2020) presents theoretical approaches to assessing multipolarity and the balance of power in the international arena. Both objective and subjective factors of perception of the balance of power in the world arena, which dominate in modern international political discourse, are analyzed. In this paper, the assessment of the potential of individual states is complemented by an analysis of strategic triangles, regional integration groupings and international alliances.

An essay by Raymond (2018) addresses the issue of multipolarity in the face of erosion of US influence. The author pays special attention to the strategy of action of third countries (on the example of the position of Australia). The work focuses on the fact that in modern discourse the problem of multipolarity is illuminated, as a rule, from the standpoint of the central role of the United States and attention is drawn to the need to take into account the legacy of the eras of colonization, decolonization, state building and the development of local norms of specific countries and regions.

The article by Blagden (2019) proposes an interpretation of the concept of "multipolarity" as a special causal concept based on the principles of the possibility of balancing by a certain group of powers of any other power, including the most powerful one. Also, this work analyzes the content and meanings invested in the terms "polarity" and "multipolarity" in modern British political discourse.

Trush (2020) provides a meaningful analysis of the prospects for Russian-Chinese political and military-political cooperation. Particular attention is paid to the risks 
and the adequacy of potential agreements from the perspective of relations in the US-China-Russia triangle.

The models considered in this work can become an effective tool for describing situations of this type and their subsequent analysis.

\section{Game-theoretic formalization of the problem of interaction of centers of power}

The main idea of the application of a game-theoretic approach to the analysis of the interaction of international centers of power in the modern political arena involves the interpretation of their relationship in the form of a cooperative game with a transferable utility $(I, v)$, where

$I=\{1, \ldots n\}-$ a set of players;

$v$ - the characteristic function $\left(2^{I} \rightarrow R\right)$, that assigns the value of the usefulness (utility, payoff) of the coalition in the event of its formation $(v(\{S\}))$ for each subset of players $S \subset I$ (a coalition of players).

Traditional concepts for solving cooperative games, such as Core, Kernel, Nucleolus, are based on the concepts of "imputation" and "pre-mputation".

The definition of "cooperative game solution" based on the concepts of "imputation" and "pre-imputation". Imputation in cooperative game $(I, v)$ is a vector $x=\left(x_{1}, x_{2}, \ldots x_{n}\right)$ satisfying the conditions:

- individual rationality

$$
x_{i} \geq v(\{i\}), \forall i \in\{1, \ldots . n\} ;
$$

- group rationality

$$
\sum_{i=1}^{n} x_{i}=v(\{I\}) .
$$

Thus, the imputation is such a distribution of the utility of the full coalition of all players (the so-called "grand coalition"), which provides each of the players with a utility no less than he can receive individually (without joining any coalition).

Within the framework of this paper, we will restrict ourselves to the models of "triple" conflicts, that is, cooperative games in which $I=\{1,2,3\}$. Both countries and intercountry associations can act as players, depending on the specific nature of the simulated situation.

The problem of choosing a methodology for constructing a characteristic function is extremely serious and fundamental from the point of view of the success or failure of the application of the game-theoretic cooperative model.

On an intuitive verbal level, the thesis that the competition between countries and blocs at the international level is for political influence seems quite reasonable. In this case, influence is understood to be the extent to which the interests of a country (or a group of countries) will be taken into account when reaching the next agreement, resolving a dispute, etc. Moreover, it is not a one-time success in a specific situation that is much more important and essential, but the accumulated weight, which determines the degree of consideration of interest in potential (future) situations. 
Unfortunately, such a verbal interpretation of "political influence" cannot be simply and unambiguously correlated with some specific and generally accepted quantitative indicators. Because of this, one has to be content with abstract (conditional) indicators.

Of course, it is very difficult to correlate such an approach with the classical interpretations of cooperative games, in which imputations and pre-imputations are treated as shares of some good (utility) in which the players are interested.

At the same time, taking into account the complexities of the real practice of using game-theoretic models, this drawback does not seem to be something particularly challenging and out of the general range of conventions and abstractions.

Within the framework of this study, the performance (utility) of players and coalitions is assessed using the abstract level of political influence, which is considered a value that takes values on the interval $[0,1] \quad(0-$ no influence, 1 - maximum influence). In the simplest situations, a quantitative assessment of the "influence" of a player (coalition) can, for example, be based on the proportion of issues (problems), the solution to which can be blocked without its consent, in the total set of international problems under consideration. While agreeing with the controversial nature of this approach, one cannot but admit that on most issues of interstate interaction, veto players are usually defined quite simply.

Here, the authors largely follow the traditions of political science applications of cooperative games laid down in the works of Shapley, Shubik (1954),Banzhaf (1965).

The paper by Konyukhovskiy, Holodkova (2017) was devoted to simple gametheoretic models of "triple conflicts".

In this paper, in particular, versions of a cooperative game with three participants were considered for various values of individual and coalition utilities. Also, the values of the main solutions for these games were obtained (Shapley vector, Core, Nucleolus). These results are of practical interest. In particular, they provide a fairly realistic and adequate interpretation of the principal trends in the development of relations in the USA - China - Russia triangle in the period from 1992 to 2016. In more general settings, one can consider not only the United States, but also the so-called "United Western World" (conditionally - "West").

For clarity and transparency of the subsequent presentation, we give a brief overview of the previously constructed models and the conclusions obtained on their basis.

Table 1 shows the versions of the characteristic functions for the "principal" interaction configurations. Hereinafter, it is assumed that player 1 is the strongest from the point of view of potential influence, then the players' opportunities decrease in the order of increasing their numbers. Based on the content of the concept of "level of influence", the value of the characteristic function for the full ("big") coalition is assumed to be equal to 1 .

Table 1. Characteristic functions for different versions of "triple interaction" models.

\begin{tabular}{|c|l|l|l|l|l|l|l|}
\hline Version & Period & $\{1\}$ & $\{2\}$ & $\{3\}$ & $\{1,2\}$ & $\{1,3\}$ & $\{2,3\}$ \\
\hline (a) & Period 1992-2008 & 0.8 & 0 & 0 & 1 & 1 & 0 \\
\hline (d) & Symmetrical multipolar world & 0 & 0 & 0 & 1 & 1 & 1 \\
\hline (e) & Asymmetrical multipolar world & 0.4 & 0.2 & 0 & 1 & 1 & 1 \\
\hline
\end{tabular}

Source:conditional data 
Model version (a) (see Table 1) corresponds to the alignment of forces in the period conventionally outlined as 1992B Ђ“"2008. At this time, there was an obvious predominance of player 1 (the "western world") and the lack of opportunities and players 2 and 3 to counterbalance his influence even in the case of joining a coalition.

Fig.1 gives a geometric interpretation of the set of non-negative pre-imputation for the game in version (a). The set of pre-imputations for the game $n=3$ is the set of vectors lying on the plane

$$
x_{1}+x_{2}+x_{3}=v(\{I\})
$$

in three-dimensional space. For these reasons, for geometric interpretations of games $n=3$, "flat illustrations" are used, representing the plane (3).

One of the simplest and most natural directions of analysis of the game in question is the study of its Core (a set of undominated imputations):

$$
C(v)=\left\{x \in R^{n} \mid(\forall S \subset I) \sum_{i \in S} x_{i} \geq v(S), \sum_{i \in I} x_{i}=v(I)\right\} .
$$

As you can see from 1 Core of game (a) is a one-point set

$$
C(v)=(1,0,0) .
$$

This fact means maximum Player 1 influence.

While recognizing the validity of possible criticism based on the arguments that the obtained solution (5) is completely determined by the degree of arbitrariness that was laid down in the construction of the characteristic function (Table 1), at the same time it should be noted that it reflects the mechanisms with a high degree of reliability the dominance of the United States (the united "West") at the stage that followed the collapse of the Soviet Union. Not having $100 \%$ influence $(v(\{1\})=0.8<1)$, Player 1 , nevertheless, ensured himself full control due to the fact that any coalitions that received $100 \%$ influence were impossible without his participation:

$$
v(\{1,2\})=v(\{1,3\})=1,
$$

provided that $v(\{2,3\})=0$.

It can also be noted that with such a structure of games, the value of the individual influence of the Player 1 is not principal. Even if it were much less than 0.8 (in the limit equal to zero), solution (5) would remain unchanged. Since it is determined solely by the fact that in the game given by Table 1 , any coalition must contain Player1 ("Union West") to be a winning one.

The thesis about the importance of transition from a unipolar world to a multipolar one is widely known. Appearing around the turn of the third millennium, it has taken one of the leading places in modern political science discourse.

One of the important positive characteristics of the proposed cooperative gametheoretic models is the possibility of describing, with their help, at a formalized level, the mechanisms of the transition from a unipolar to a multipolar world order. The latter correspond to model versions (d) and (e), in Table 1.

Model version (d) reflects a situation in which no player alone can gain influence in the world. At the same time, any coalition of two participants gains absolute 


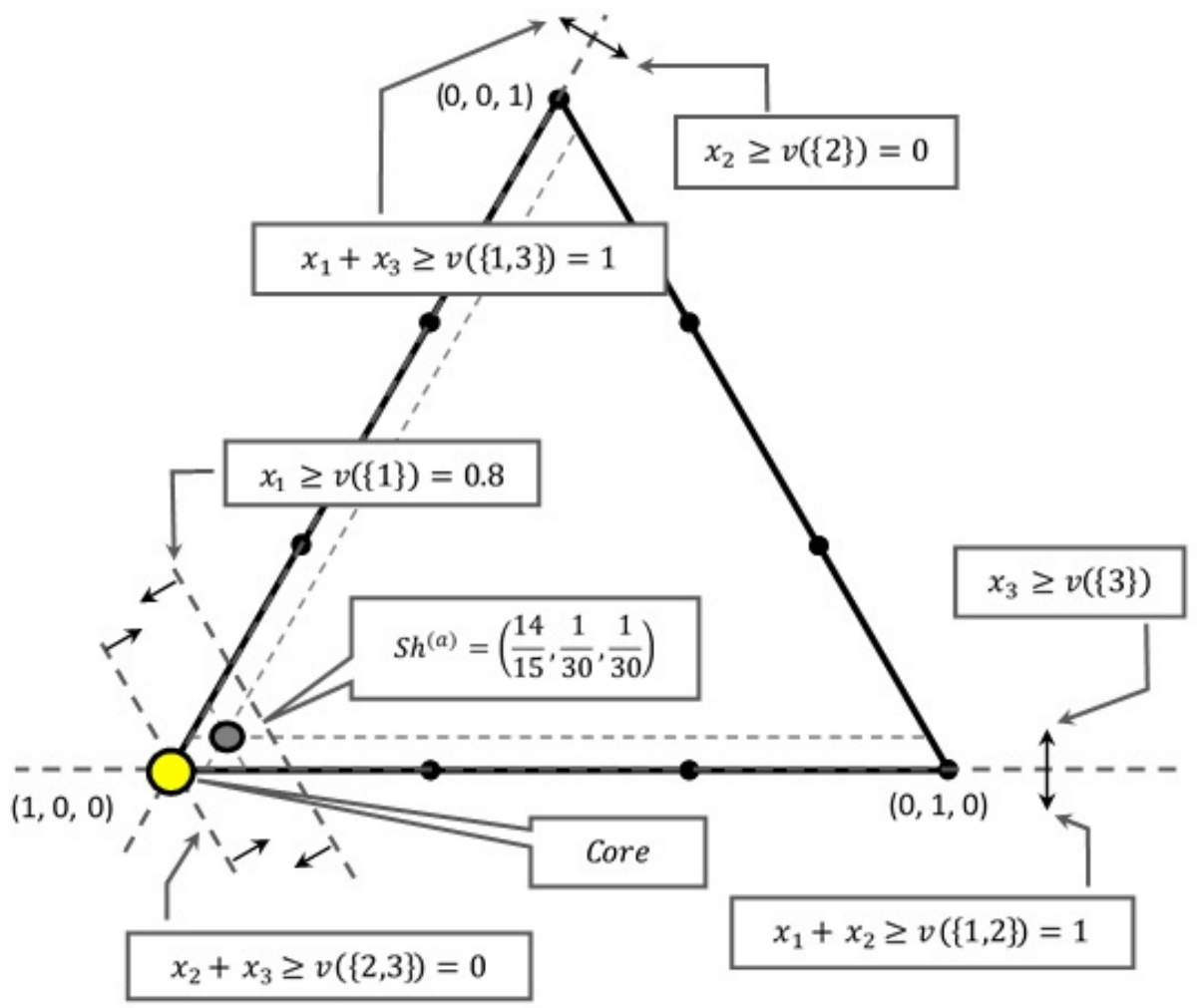

Fig. 1. Geometric interpretation of the set of pre-divisions for the game, version (a)

influence (it can impose its own conditions of the game on a third non-aligned participant). Of course, the full coalition also has absolute influence.

A geometric illustration of the set of pre-imputations for the game in version (d) is shown in Fig.2

As you can see, the game in version (d) has an empty core. The intersection of the lines of coalition rationality gives us three "principle" points

$$
x^{1,2}=\left(\frac{1}{2}, \frac{1}{2}, 0\right), x^{1,3}=\left(\frac{1}{2}, 0, \frac{1}{2}\right), x^{2,3}=\left(0, \frac{1}{2}, \frac{1}{2}\right)
$$

Each of them corresponds to a "conspiracy" of two players who share the influence equally, with the exclusion of the third participant who receives nothing. These distributions are characterized by obvious instability, since there are obvious threats against them. With any configuration, each of the "united parties" has reason to suspect the partner that he can, without loss of usefulness for himself, agree with the "superfluous third." Nucleolus in game (d), due to the symmetry of the participants' capabilities, is the vector

$$
N^{(d)}=\left(\frac{1}{3}, \frac{1}{3}, \frac{1}{3}\right)
$$




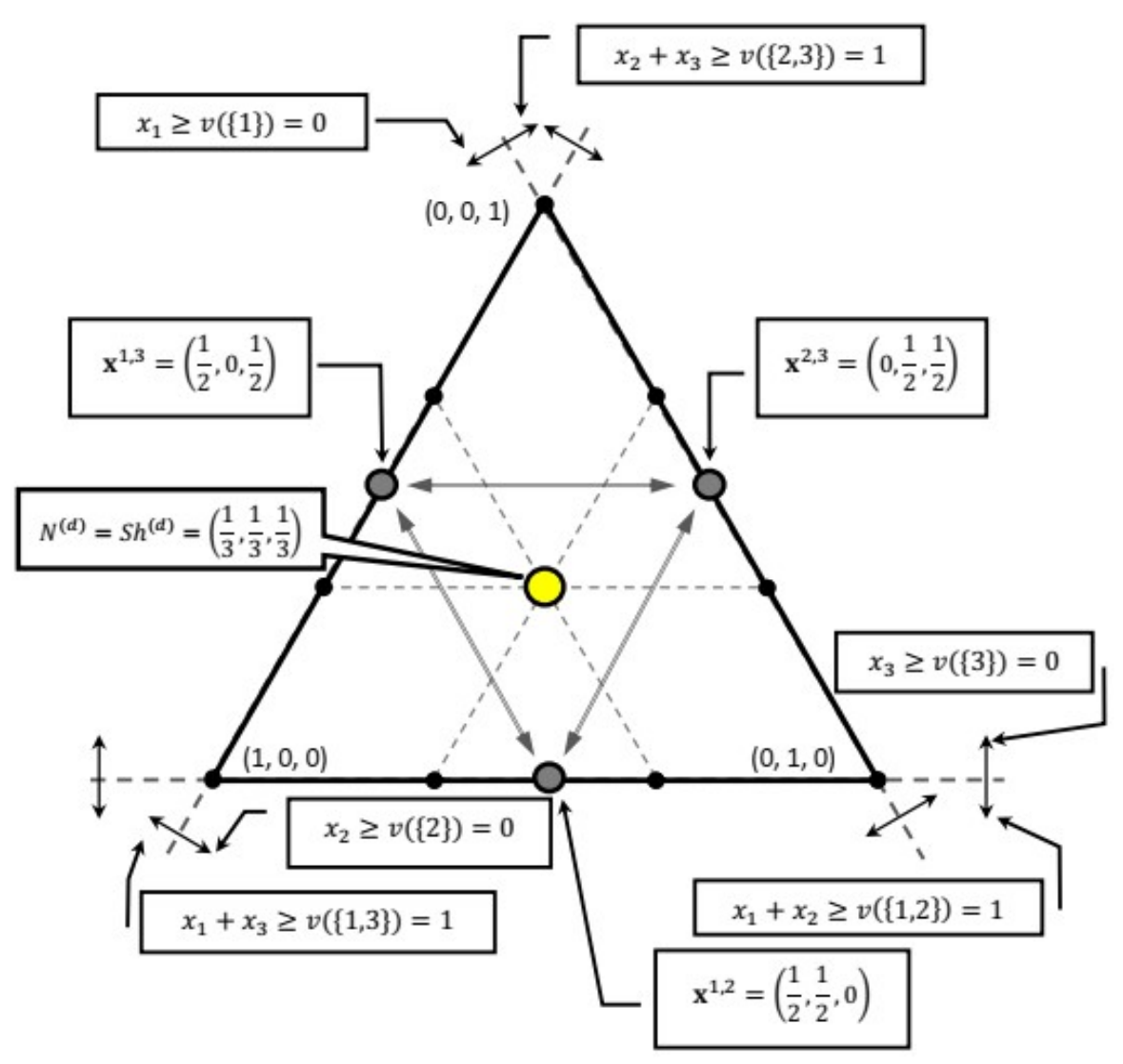

Fig. 2. Geometric interpretation of the set of pre-divisions for the game, version (d)

It is easy to check that the Nucleolus in this game coincides with the Shapley vector. However, (6) has all the disadvantages of an "unstable, contested" solution, from which the players have incentives to deviate (going between $x^{1,2}, x^{1,3}$ and $x^{2,3}$ ).

The "symmetry" of the instability of the solutions $x^{1,2}, x^{1,3}, x^{2,3}$ in the game (d) is due to the complete symmetry of the players' capabilities. The realism of such an assumption, obviously, raises serious doubts. This disadvantage can be partly overcome by differentiating the individual utilities of the players - version (e), Tabel 1.

The characteristic function in model (e) is based on the assumption of the relative increase in the potential of player 2 in relation to player 3 . Of course, the ratio itself is 0.2 to 0.4 can become a reason for disputes and objections. However, at the level of qualitative analysis, one can agree with such values. First of all, because it is not so much the absolute values as their ordering relative to each other that are of fundamental importance.

Fig.3 (a geometric illustration of the game (e)) allows us to visually demonstrate the transformation of the solutions $x^{1,2}, x^{1,3}, x^{2,3}$, which we identified in the game (d) and assuming the distribution of influence between two players when excluding 


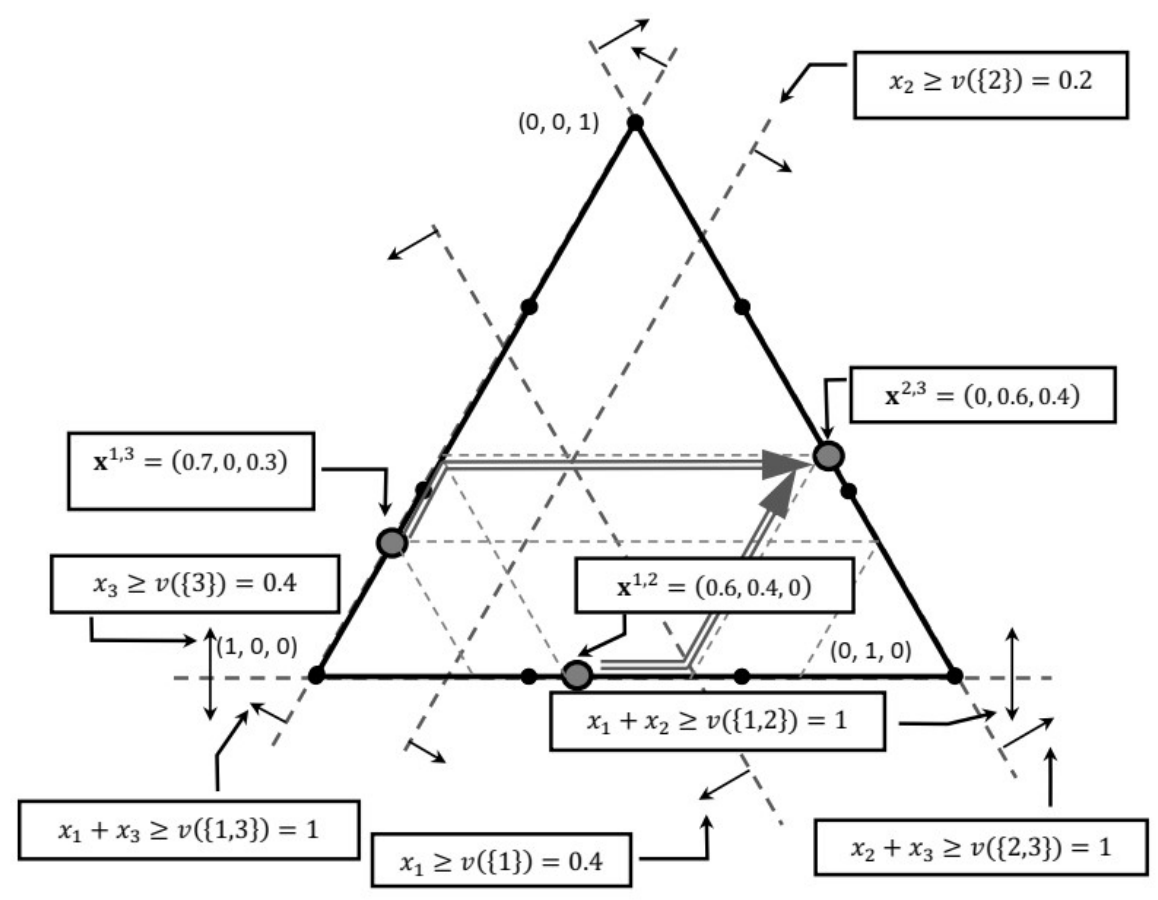

Fig. 3. Geometric interpretation of the set of pre-divisions for the game, version (e) 
from the division third party. It is assumed that the beneficiary players $(i, j)$ in the division $x^{i, j}$ equally divide the value $v(\{I\})-v(\{i\})-v(\{j\})$, i.e. the difference between the usefulness of a full coalition (maximum possible impact) and their individual usefulness. For example,

$$
\begin{gathered}
x^{1,2}=\left(v(\{1\})+\frac{v(\{I\})-v(\{1\})-v(\{2\})}{2}, v(\{2\})+\frac{v(\{I\})-v(\{1\})-v(\{2\})}{2}, 0\right)= \\
x^{1,2}=\left(0.4+\frac{1-0.4-0.2}{2}, 0.2+\frac{1-0.4-0.2}{2}, 0\right)=(0.6,0.4,0) .
\end{gathered}
$$

As it is easy to see, in game (e), participants 2 and 3 , if they "achieve" the distribution $x^{2,3}=(0,0.6,0.4)$, have no incentive to abandon this alliance in favor of coalitions with Player 1, in which they gets a smaller share.

In a sense, the higher individual utility of Player 1, and hence the higher level of "initial grievances," work against him. They make a separate agreement with him less profitable for other participants in the game. Another important consequence of the differences between models (d) and (e) is that in model (e) Nucleolus differs from the Shapley vector. The essential differences between games (d) and (e) quite clearly demonstrate the differences that arise between the Nucleolus. By comparing different concepts of solutions in the framework of model (e), it is possible to formulate proposals for a consistent distribution in case the players overcome the "temptation of pairing" and are able to form a "big coalition":

Player 1 - from 33 to $43 \%$ influence;

Player 2 - about $33 \%$ influence;

Player 3 - from 23 to $33 \%$.

\section{Bargaining set concept}

Studies of triple models of opposition of centers of power leads us to the conclusion that the situation is typical, in which there is no expediency in the formation of a full coalition of participants (i.e. the utility of the full coalition is set to zero). In such cases, the bargaining set is a more preferable and adequate solution concept (in comparison with Core, Nucleolus, Shapley vector). This concept was proposed in a fundamental article by Aumann, Maschler (1961).

For a brief introduction, we have to introduce the tern of payoff configuration

$$
(x, \mathbf{S})=\left(x_{1}, x_{2}, \ldots, x_{n} ; S^{1}, S^{2}, \ldots, S^{m}\right),
$$

where $x_{1}, x_{2}, \mathrm{~B} \mathrm{~T}_{1} x_{n}$ - the amount of utility received by players.

$S^{1}, S^{2}, \ldots, S^{m}$ - a set of coalitions in which players unite to achieve the specified utility values. This set of coalitions must satisfy for the next conditions:

- the condition of pairwise empty intersection of coalitions

$$
S^{i} \cap S^{k}=\varnothing, i \neq k
$$

- the completeness of the set 


$$
\bigcup_{i=1}^{n} S_{i}=I
$$

- reachability of the promised distribution for each coalition

$$
\sum_{k \in S^{i}} x_{k} \leq v\left(\left\{S^{i}\right\}\right)
$$

The idea embodied in the definition of payoff configuration is quite transparent and simple. The players are divided into the coalition system $\mathbf{S}=\left(S^{1}, S^{2}, S^{m}\right)$, each coalition achieves some result (utility) $v\left(\left\{S^{i}\right\}\right)$, which, in turn, is divided between its members in accordance with the shares given by the vector $x=\left(x_{1}, x_{2}, x_{n}\right)$.

The natural requirement for a payoff configuration seems to be the conditions of coalition rationality $(\mathrm{CR})$

$$
\left(\forall S, S^{i} \in\{\mathbf{S}\}, S \subset S^{i}\right) \sum_{k \in S} x_{k} \geq v(\{S\}) .
$$

The condition (11) means that a rational payoff configuration across all its coalitions must ensure that any subsets of players (sub-coalitions) included in these coalitions are paid no less than those that are available to these sub-coalitions independently (without joining other coalitions).

Let $K$ be some coalition of participants $(K \subset I)$. Player $j$ is called a partner $K$ in the payoff configuration $(x, \mathbf{S})$.

If he is a member of such a coalition $S^{i} \in\{\mathbf{S}\}$ such that $K \cap S^{i} \neq \varnothing$. Denote by $P[K ;(x, \mathbf{S})]$ the set of all partners of the coalition $K$ in the payoff configuration $(x, \mathbf{S})$, i.e.

$$
P[K ;(x, \mathbf{S})]=\left\{j \mid j \in S^{i}, K \cap S^{i} \neq \varnothing\right\} .
$$

Pay attention to the following important points.

First, in accordance with the proposed definition, a player who is a partner of the coalition $\mathrm{K}$ may not belong to this coalition.

It is enough that he belongs to a coalition, the individual players of which belong to $K$. Secondly, $K \subset P[K ;(x, \mathbf{S})]$.

The latter is quite obvious: every member of $K$ is the partner for other participants of this coalition, certainly.

Let $(x, \mathbf{S})$ be a coalitionally rational payoff configuration in some cooperative game $\Gamma$. Let $K$ and $L$ be disjoint subsets of some coalition $S^{i} \in\{\mathbf{S}\}\left(K \subset S^{i}, L \subset\right.$ $\left.S^{i}, K \cap S^{i}=\varnothing\right)$.

Then the objection of coalition $K$ against coalition $L$ in payoff configuration $(x, \mathbf{S})$ will be called the coalition-rational payoff configuration

$$
(y, \mathbf{C})=\left(y_{1}, y_{2}, \ldots, y_{n} ; C^{1}, C^{2}, \ldots, C^{l}\right),
$$


for which

$$
\begin{gathered}
P[K ;(y, \mathbf{C})] \cap L=\varnothing, \\
(\forall j \in K) y_{j}>x_{j}, \\
\forall(j \in P[K ;(y, \mathbf{C})]) y_{j} \geq x_{j} .
\end{gathered}
$$

The conditions (12)-(14) mean that players belonging to coalition $K$, without participation of players belonging to coalition $L$, within a certain coalition-rational payoff configuration $(y, \mathbf{C})$ (different from the current payoff configuration $(x, \mathbf{S})$ ) can get better results. At the same time, the reasonability of the change of configurations is confirmed by the fact that their "new partners" will receive payments in $(y, \mathbf{C})$ no less than in $(x, \mathbf{S})$.

Let $(x, \mathbf{S})$ be the coalitionally rational payoff configuration in the game $\Gamma,(y, \mathbf{C})$ be the objection of the coalition $K$ against the coalition $L$ with respect to $(x, \mathbf{S}), K \subset$ $S^{i}, L \subset S^{i}, K \cap S^{i}=\varnothing$. The counter objection of the coalition $L$ against the coalition $K$ will be called the coalitionally rational payoff-configuration.

$$
(z, \mathbf{D})=\left(z_{1}, z_{2}, \ldots z_{n} ; D^{1}, D^{2}, \ldots D^{k}\right)
$$

for which

$$
\begin{gathered}
K \not \subset P[L ;(z, \mathbf{D})] \\
(\forall j \in P[L ;(z, \mathbf{D})]) z_{j} \geq x_{j}, \\
(\forall j \in P[L ;(z, \mathbf{D})] \cap P[K ;(y, \mathbf{C})]) z_{j} \geq y_{j} .
\end{gathered}
$$

The conditions (15)-(17) mean that the coalition $L$ in response to the "threatening" payoff configuration $(y, \mathbf{C})$ can offer a payoff configuration $(z, \mathbf{D})$ in which its members, its new partners, and potential coalition partners $\mathrm{K}$ by configuration $(y, \mathbf{C})$ will receive a payment not less than in the current configuration $(x, \mathbf{S})$. At the same time to achieve this result, she does not need members of the coalition $K$.

A coalitionally rational payoff configuration $(x, \mathbf{S})$ is called stable if for each objection of $K$ against $L$ there is counter-objection $L$ against $K$. The set of all stable coalitionally rational payoff configurations in the game $\Gamma$ will be called the bargaining set ( $\mu$-set or $\mu$-stable set). The following theorem is valid.

The bargaining set of a game $\Gamma$ can be represented as a set of solutions of a conjunctive-disjunctive linear inequality system with respect to variables $x_{j}$.

Thus, the bargaining set can be represented as the union of convex polyhedrals in $n$-dimensional linear space.

For the case of a game with three participants and utilities given by Table 2 the expression for the distributions of the payoff configurations of the participants has a fairly simple and understandable form.

In accordance with the definitions (12)-(14), (15)-(17) the bargaining set of the game given in Table 2 belongs to the payoff configuration $((0,0,0) ;\{1,2,3\})$. 
Table 2. Utilities for the game $n=3$ with nonzero utilities of paired coalitions

\begin{tabular}{|l|l|l|l|l|l|l|}
\hline$\{1\}$ & $\{2\}$ & $\{3\}$ & $\{1,2\}$ & $\{1,3\}$ & $\{2,3\}$ & $\{1,2,3\}$ \\
\hline 0 & 0 & 0 & $v_{1,2}$ & $v_{1.3}$ & $v_{2,3}$ & 0 \\
\hline
\end{tabular}

Source:conditional data

If there is no "dominant" pair coalition, that is, if the coalition utilities satisfy the inequalities

$$
v_{12} \leq v_{13}+v_{23}, \quad v_{13} \leq v_{12}+v_{23}, \quad v_{23} \leq v_{12}+v_{13}
$$

then the payoff configurations forming the bargaining set have the form

$$
\left\{\begin{array}{l}
x_{1}=\frac{v_{12}+v_{13}-v_{23}}{2}, x_{2}=\frac{v_{12}+v_{23}-v_{13}}{2}, x_{3}=0 ;\{1,2\},\{3\} \\
x_{1}=\frac{v_{12}+v_{13}-v_{23}}{2}, x_{2}=0, x_{3}=\frac{v_{13}+v_{23}-v_{12}}{2} ;\{1,3\},\{2\} \\
x_{1}=0, x_{2}=\frac{v_{12}+v_{23}-v_{13}}{2}, x_{3}=\frac{v_{13}+v_{23}-v_{12}}{2} ;\{1\},\{2,3\} .
\end{array}\right.
$$

In the case of the paired coalition $\{1,2\}$, the objection of player 1 against player 2 means the transition to the coalition $\{1,3\}$, and the counter-objection 2 against 1 means the transition to the coalition $\{2,3\}$. Thus, it makes no sense for player 1 to "destroy" the coalition $\{1,2\}$ if the share he receives in alliance with player 2 is not less than the share that player 3 can offer

$$
x_{1}=v_{12}-x_{2} \geq v_{13}-x_{3} .
$$

At the same time, the player 2 will support the coalition $\{1,2\}$ if the share he receives in alliance with player 1 is also not less than the share that player 3 can offer

$$
x_{2}=v_{12}-x_{1} \geq v_{23}-x_{3}
$$

and of course the distribution $x_{1}+x_{2}$ should correspond to the abilities of the coalition

$$
x_{1}+x_{2}=v_{12} .
$$

Repeating similar reasoning for other coalitions (taking into account possible objections and counter- objections), we come to the conclusion that conditions (20)(21) must be satisfied as strict equalities

$$
v_{12}-x_{2}=v_{13}-x_{3}, v_{12}-x_{1}=v_{23}-x_{3} .
$$

Whence, taking into account (22), an explicit expression for $x_{3}$ is obtained. Explicit expressions for $x_{1}$ and $x_{2}$ are obtained in a similar way.

In Fig. 4 shows a geometric illustration of the bargaining set for the game under consideration (Table 2), in the absence of a prevailing (dominant) pair coalition.

If there is a "dominant" pair coalition (for example, $v_{12}>v_{13}+v_{23}$ ), then the payoff configurations forming the bargaining set have the form 


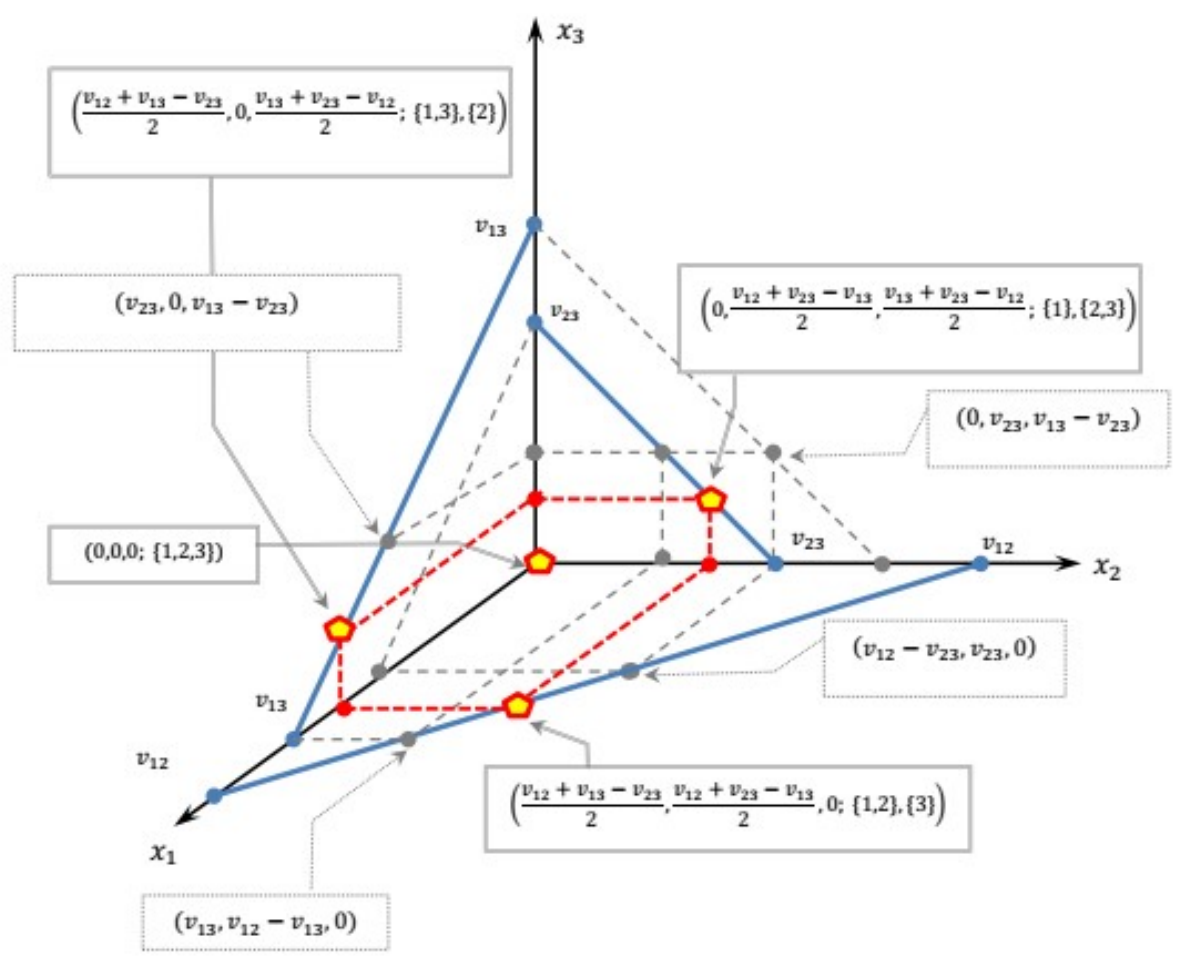

Fig. 4. Bargaining set for the situation of the absence of a dominant pair coalition: $v_{12} \leq v_{13}+v_{23}, v_{13} \leq v_{12}+v_{23}, v_{23} \leq v_{12}+v_{13}$

$$
\left\{\begin{array}{l}
v_{13} \leq x_{1} \leq v_{12}-v_{23}, x_{2}=v_{12}-x_{1}, x_{3}=0 ;\{1,2\},\{3\} \\
x_{1}=v_{13}, x_{2}=0, x_{3}=0 ;\{1,3\},\{2\} \\
x_{1}=0, x_{2}=v_{23}, x_{3}=0 ;\{1\},\{2,3\} .
\end{array}\right.
$$

The geometric illustration of the bargaining set for the case of the existence of a prevailing coalition $\left(v_{12}>v_{13}+v_{23}\right)$ is given in Fig.5.

A rigorous derivation of formulas (19) and (24) specifying payoff configurations for the game under consideration is given in the work in Auman - Mashler.

From the point of view of potential applied aspects of this model, the direction of research of transformation points of specific characteristic functions, at which the configuration of solutions changes from (19) to (24) and vice versa, seems promising and interesting.

It is obvious that such a transformation in real triple conflicts radically changes the system of relationships between the participants, sharply strengthening the "strongest" and actually nullifying the opportunities and claims of the "weak partners".

In what follows, by default (without loss of generality), we can accept the assumption that the players and coalitions are numbered in such a way that the relations 


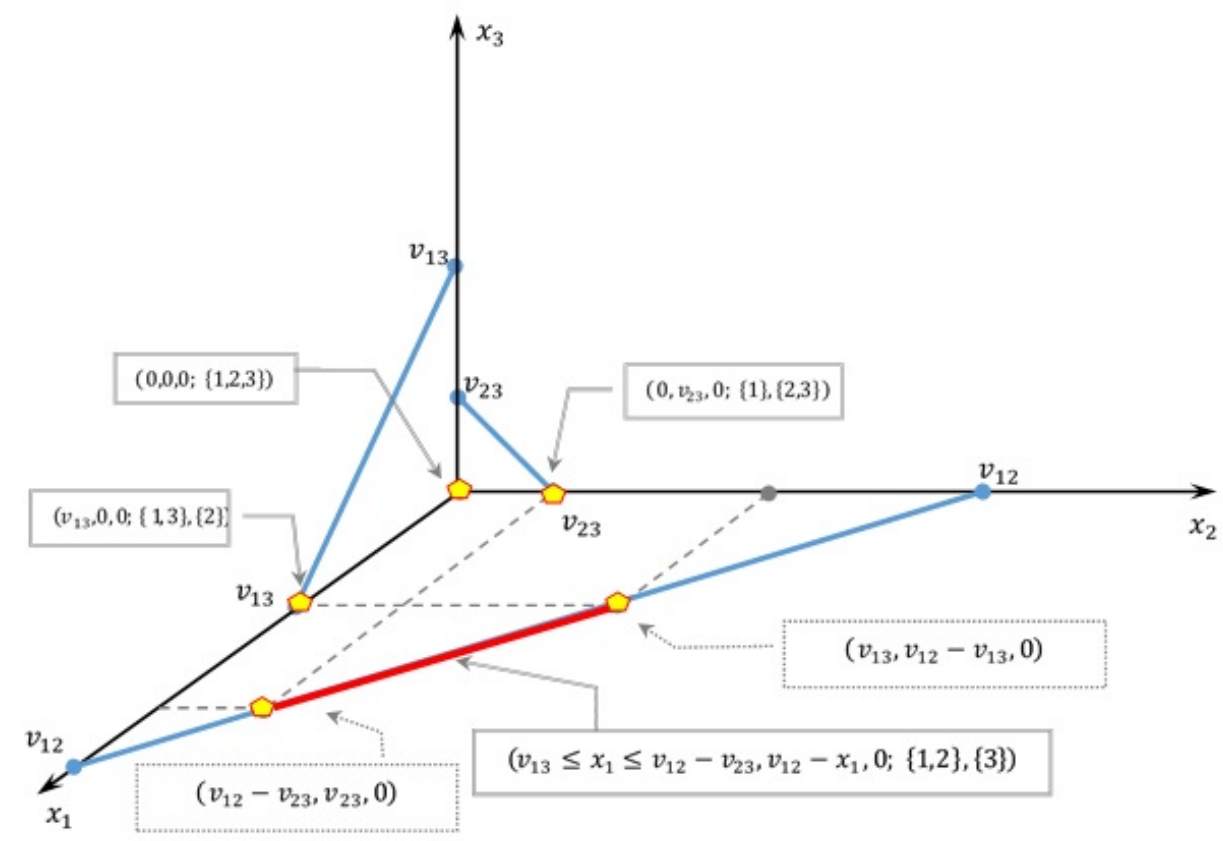

Fig. 5. Bargaining set for the situation of the existence of a dominant pair coalition: $v_{12}>v_{13}+v_{23}$

$$
v_{1,2} \geq v_{1,3} \geq v_{2,3},
$$

i.e. there is a gradual decrease in the strength of coalitions from $\{1,2\}$ to $\{2,3\}$ and from the point of view of coalition utility player 1 is the strongest, and player 3 , on the contrary, is the weakest.

\section{Stochastic cooperative games}

One of the problems that we face when used as a base for the construction of the characteristic function of the minimum income in the currency volatility, built on the basis of a portfolio of exchange members of the coalition is the stochastic nature of the data. A constructive solution to this problem is associated with the transition from deterministic to stochastic cooperative games.

In the modern theory of cooperative games it has developed several approaches to the definition of "stochastic cooperative game". One of the first studies in this direction was the work Charnes, Granot (1973) Charnes, Granot (1977). Also worth mentioning is the series of works on the subject Yeung, Petrosyan (2004), Yeung, Petrosyan (2006), Suijs, Born (1999),Suijs (1999), Suijs, Born (1999).

In this article a stochastic cooperative game (SCG) we mean a pair $\Gamma=(I, \tilde{v})$ where

$I=\{1 \ldots n\}-$ the set of participants;

$\tilde{v}(S)$ - random variables with known distribution density $p_{\tilde{v}(S)}(x)$ which interpreted as revenues (utility payments), the corresponding coalitions $S \subset I$.

This approach to the task of stochastic cooperative games were previously presented in the paper Konyukhovskiy (2012). 
Imputation stochastic cooperative game will be called the vector $x(\alpha) \in R^{n}$ satisfying for conditions

$$
\text { (a) }(\forall i \in I) P\left\{x_{i}(\alpha) \geq \tilde{v}(i)\right\} \geq \alpha
$$

- stochastic analog of individual rationality;

$$
\text { (b) } P\left\{\sum_{i=1}^{n} x_{i}(\alpha) \leq \tilde{v}(I)\right\} \geq \alpha
$$

- stochastic analog of the group rationality.

Note that the condition (26) sets that the share prescribed by delay $x(\alpha)$ for $\mathrm{i}$-th player has to be greater or equal than the random value of his personal gain with a probability of not less than $\alpha$. In accordance with (26) i-th component of the vector division $x(\alpha)$ compared with the $\alpha$-quantile $F_{\tilde{v}(i)}(x)$ (distribution function of the random variable $\tilde{v}(i)$ ). For compactness subsequent expressions we introduce the notation

$$
v_{\alpha}(i)=F_{\tilde{v}(i)}^{-1}(\alpha)
$$

for some $i-t h$ player,

$$
v_{\alpha}(S)=F_{\tilde{v}(S)}^{-1}(\alpha)
$$

for some coalition $S \subset I$. Then (26) can be written as

$$
(\forall i \in I) x_{i}(\alpha) \geq v_{\alpha}(i) .
$$

The possibility of transformation from condition (26) to (30) follows from the properties of non-decreasing distribution functions. Indeed, the condition $x_{i}(\alpha) \geq$ $\tilde{v}(i)$ is satisfied for a certain level of probability $\alpha$, will be carried out for all $\alpha^{\prime}>\alpha$.

In the classical cooperative games under group rationality means the need for full distribution utility large (complete) coalition within the division. In a modification of the stochastic game (27) means that the big (full) coalition is able to win with a probability of not less than $\alpha$, to ensure the realization of the imputation $x(\alpha)$ Note that the condition (27) is equivalent to

$$
P\left\{\sum_{i=1}^{n} x_{i}(\alpha) \geq \tilde{v}(I)\right\} \leq 1-\alpha .
$$

From (31), denoting $v_{\alpha}(I)=F_{\tilde{v}(I)}^{-1}(\alpha)$ the $\alpha$-quantile of the distribution function $F_{\tilde{v}(I)}(x)$, we obtain $\sum_{i=1}^{m} x_{i}(\alpha) \geq v_{1-\alpha}(I)$.

We emphasize quite a significant difference. If the normal condition of cooperative games group rationality is defined as the strict equality and thus defines a hyperplane in $n$-dimensional space, the approach proposed here it is in the form of inequality and defines loosely in half-dimensional space. Thus, the "nature" of vectors that satisfy the definition (26)-(27) differs from the "nature" of imputations in their classical interpretation. Sometimes for naming such objects use the term "distributions" (allocations). 
As a result, the system conditions, which determines imputation stochastic game, takes the form

$$
\begin{aligned}
& \text { (a) }(i \in I) x_{i}(\alpha) \geq v_{\alpha}(i), \\
& \text { (b) } \sum_{i=1}^{n} x_{i}(\alpha) \leq v_{1-\alpha}(I) .
\end{aligned}
$$

For values $v_{\alpha}(i)$ in modern risk management usually apply the term value at risk VaR (Value at Risk). In this connection it may be noted advantages of the approach (32)-(33). Namely, it is logically consistent with the concept of VaR. This potentially opens up opportunities for a meaningful interpretation of the results of subsequent studies of the properties of this class of games and concepts of finding solutions.

\section{Implementation of the concept of a negotiation set on stochastic cooperative models}

As it is easy to see, the assumption about the deterministic nature of the utility (payoffs) of coalitions $v_{i, j}$ is rather rigid. In reality, it is extremely difficult to unequivocally assess the consequences and effects of coalition associations, especially given the hypothetical nature of some of them.

As a consequence, cooperative game models with stochastic characteristic functions are more promising and preferable in the case of interaction of international centers of power, where each coalition of players $S \subset 2^{I}$ is associated with a random variable $\tilde{v}_{\{S\}}$ (random utility) with some known distribution density $p_{\tilde{v}_{\{S\}}}(x)$.

In the case of stochastic game models, there is a transformation of the relationships reflecting the system of mutual objections and counter-objections.

In particular, the objections of player 1 against player 2 consists in abandoning the coalition $\{1,2\}$ and creating the coalition $\{1,3\}$, will be justified if the inequality

$$
\tilde{v}_{1,2}-x_{2} \leq \tilde{v}_{1,3}-x_{3}
$$

that is, provided that the share of the first player does not decrease.

On the other hand, the "compensating" threat of player 1 against player 3 (abandoning the coalition $\{1,3\}$ and forming a coalition with player 2 ) leads to the opposite inequality

$$
\tilde{v}_{1,3}-x_{3} \leq \tilde{v}_{1,2}-x_{2},
$$

It is as a result of combining such inequalities in the deterministic case that the equations that determine the bargaining set are obtained. It is easy to see that this approach cannot be directly extended to the stochastic version of the game, so for continuous random variables (as instance, $\tilde{v}_{1,2}, \tilde{v}_{1,3}$ )

$$
\operatorname{Pr}\left\{\tilde{v}_{1,2}-x_{2}=\tilde{v}_{1,3}-x_{3}\right\}=0 .
$$

In this regard, an approach based on determining the values of $x_{2}$ and $x_{3}$ from the condition of equiprobability of events seems to be constructive

$$
x_{2}-x_{3} \leq \tilde{v}_{1,2}-\tilde{v}_{1,3} \text { and } x_{2}-x_{3} \geq \tilde{v}_{1,2}-\tilde{v}_{1,3}
$$


or

$$
\operatorname{Pr}\left\{\tilde{v}_{1,2}-\tilde{v}_{1,3} \leq x_{2}-x_{3}\right\}=\operatorname{Pr}\left\{\tilde{v}_{1,2}-\tilde{v}_{1,3} \leq x_{2}-x_{3}\right\}=0.5 .
$$

In terms of content, condition (38) means the choice of the median value of the random variable $\tilde{v}_{1,2}-\tilde{v}_{1,3}$ as an estimate for the difference $x_{2}-x_{3}$.

For compactness in the subsequent presentation we will use the notation

$$
\tilde{w}_{k, i}=\tilde{v}_{k, i}-\tilde{v}_{k, j}, i, j, k \in\{1,2,3\} .
$$

Accordingly, $p_{\tilde{w}_{i, j}}(x)$ is the density function of the random variable $\tilde{w}_{i, j}$, and $F_{w_{\tilde{i}, j}}(x)=\operatorname{Pr}\left\{\tilde{w}_{i, j} \leq x\right\}$ is the distribution function of $\left.\tilde{w}_{i, j}, F_{\tilde{w}_{i, j}}^{-1}(x)\right)$ is the inverse distribution function.

Based on this logic, we can obtain similar expressions for the remaining pairs of mutual objections and counter- objections. As a result, we get

$$
x_{i}-x_{j}=\operatorname{median}\left(\tilde{v}_{k, i}-\tilde{v}_{k, j}\right), i, j, k \in\{1,2,3\} .
$$

We also denote

$$
\left.\operatorname{median}\left(\tilde{v}_{k, i}-\tilde{v}_{k, j}\right)\right)=m_{i, j}, i, j, k \in\{1,2,3\} .
$$

Based on (40) (for specific $i, j, k$ ), we can obtain the relations

$$
\begin{aligned}
& x_{1}-x_{2}=m_{1,2}=F_{\tilde{w}_{1,2}}^{-1}(0.5), \\
& x_{1}-x_{3}=m_{1,3}=F_{\tilde{w}_{1,3}}^{-1}(0.5)
\end{aligned}
$$

this allows expressing

$$
m_{1,2}+m_{1,3}=x_{1}-x_{2}+x_{1}-x_{3}=2 x_{1}-\left(x_{2}+x_{3}\right)
$$

and further

$$
x_{1}=\frac{\left(m_{1,2}+m_{1,3}\right)+\left(x_{2}+x_{3}\right)}{2} .
$$

By analogy from expressions

$$
\begin{aligned}
& x_{2}-x_{1}=m_{2,1}=F_{\tilde{w}_{2,1}}^{-1}(0.5), \\
& x_{2}-x_{3}=m_{2,3}=F_{\tilde{w}_{2,3}}^{-1}(0.5)
\end{aligned}
$$

we can get

$$
x_{2}=\frac{\left(m_{2,1}+m_{2,3}\right)+\left(x_{1}+x_{3}\right)}{2}
$$

and

$$
x_{3}=\frac{\left(m_{3,1}+m_{3,2}\right)+\left(x_{1}+x_{2}\right)}{2} .
$$

In the deterministic version of the game, the single-valued values $x_{k}$, were obtained by substituting the values of the utilities of pairwise coalitions $\left(x_{i}+x_{j}=v_{i, j}\right)$. 
In the case of stochastic coalitional utilities, an approach seems reasonable, according to which the deterministic coalitional utility is replaced by its estimate based on the principles of the $\mathrm{VaR}$ (value at risk) concept (for a certain given level of probability $\alpha$ ).

With this approach, the utility that a coalition of players $i, j$ can "reasonably" claim is estimated by the $(1-\alpha)$-quantile of the distribution function of the random variable $\tilde{v}_{i, j}$.

$$
v_{i, j}^{(1-\alpha)}=F_{\tilde{v}_{i, j}}^{-1}(1-\alpha)
$$

In terms of content, this means that if

$$
x_{i}+x_{j}=v_{i, j}^{1-\alpha}=F_{\tilde{v}_{i, j}}^{-1}(1-\alpha)
$$

then

$$
\operatorname{Pr}\left\{x_{i}+x_{j} \leq \tilde{v}_{i, j}\right\} \geq \alpha,
$$

i.e. with probability at least $\alpha$, the utility of the coalition $\{i, j\}$ will provide the payoff $x_{i}+x_{j}$. In this case, the values of the distributions belonging to the stochastic bargaining set are given as

$$
x_{k}=\frac{\left.\left(m_{k, i}+m_{k, j}\right)+v_{i, j}^{(1-\alpha)}\right)}{2} .
$$

By analogy with (19), a stochastic bargaining set can be described by a system of payoff configurations

$$
\left\{\begin{array}{l}
x_{1}, x_{2}, 0 ;\{1,2\},\{3\} \\
x_{1}, 0, x_{3} ;\{1,3\},\{2\} \\
0, x_{2}, x_{3} ;\{1\},\{2,3\}
\end{array}\right.
$$

where the value $x_{1}, x_{2}, x_{3}$ are determined by the formulas (53).

A significant warning must be given. The stochastic nature of the model also predetermines the hypothetical nature of the values of the payoffs $x_{k}$ given by formulas (53).

In other words, they should be considered as some potential basis for reaching coalition agreements when the coalition members realize the zero probability of obtaining utility exactly equal to the values from formulas (53).

\section{Empirical aspects of the analysis of stochastic cooperative models of interaction of centers of force}

Let us dwell on some aspects of the practical application of the bargaining set concept for the stochastic implementation of a cooperative game with three participants.

First of all, we need to decide on the choice of the class of distribution density functions used in modeling the stochastic utilities of players. Based on the meaningful properties of the models describing the processes of interaction of world centers of power, the "acceptable" candidates are, for example, asymmetric triangular distributions. 
The corresponding graphs are shown in Fig.6 (density functions), Fig.7 (distribution functions).

\section{Triangular density functions}

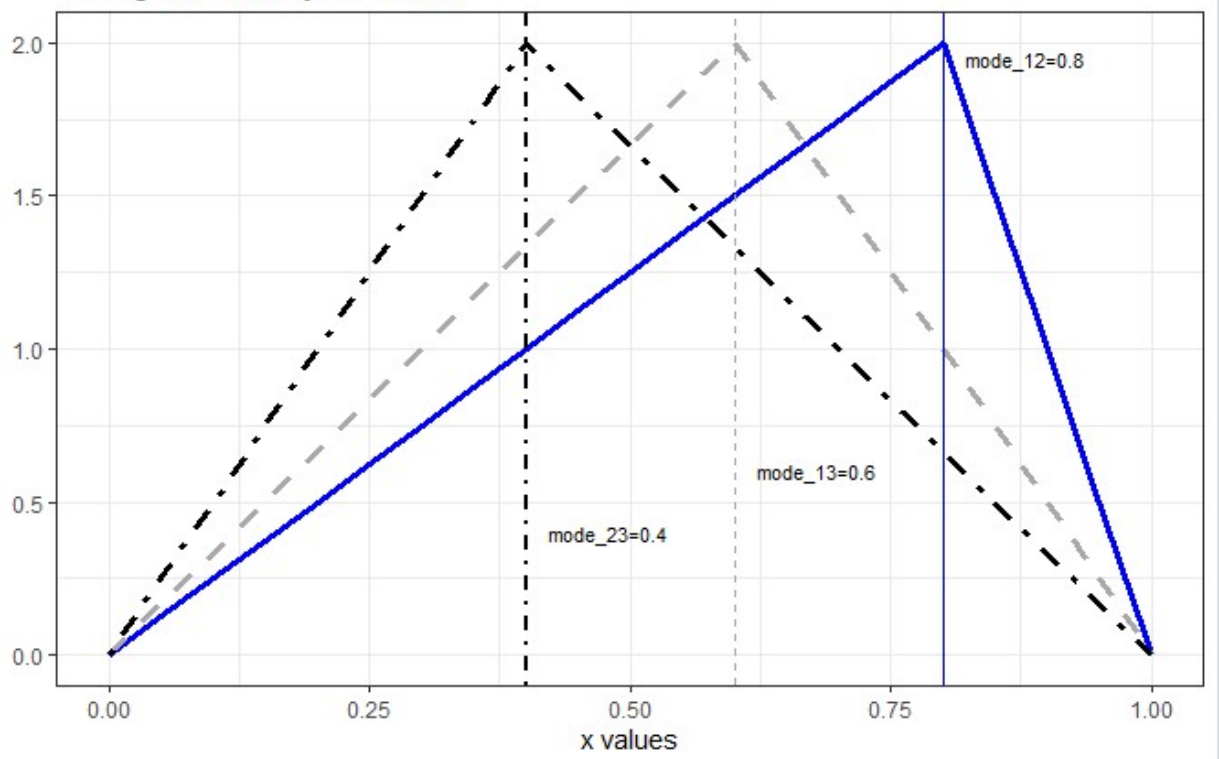

Fig. 6. Example of density for the asymmetrical triangular distribution functions (for stochastic coalition utilities $\tilde{v}_{1,2}, \tilde{v}_{1,3}, \tilde{v}_{2,3}$

Another quite suitable distribution for the models we are considering is the distributions of the PERT class. The corresponding graphs are shown in Fig.8 (density functions) Fig.9 (distribution functions).

The density and distribution functions for the differences $\tilde{w}_{k, j}$ determined by formulas (39) do not have simple explicit expressions. This is true both in the case of the distribution of pairwise coalition utilities $\tilde{v}_{i, j}$ according to the asymmetric triangular law, and in the case of their distribution according to the PERT law. For this reason, methods based on numerical modeling of stochastic differences $\tilde{w}_{k, j}$ seem to be promising. Let us give examples of constructing stochastic bargaining sets for the case of PERT distribution of pairwise coalition utilities.

Provided that all utilities in the game are normalized and take values on the interval $[0,1]$, the PERT density is uniquely determined by the choice of the mode.

Table 3 presents two variants of a stochastic cooperative game that simulates the conditions for the interaction of centers of power.

Table 4 shows the values of the components $x_{i}(i=\{1,2,3\})$ forming a stochastic bargaining set for two principal versions of pairwise coalition utilities, at different levels of probability $\alpha$.

Negative values $x_{3}$, in the rows of Table 4 , in terms of meaning, can be interpreted as an additional reason for doubting the possibility of coalitions with the participation of a third player and, accordingly, as a low assessment of his ability to influence (within the modeling situation). 


\section{Triangular distribution functions}

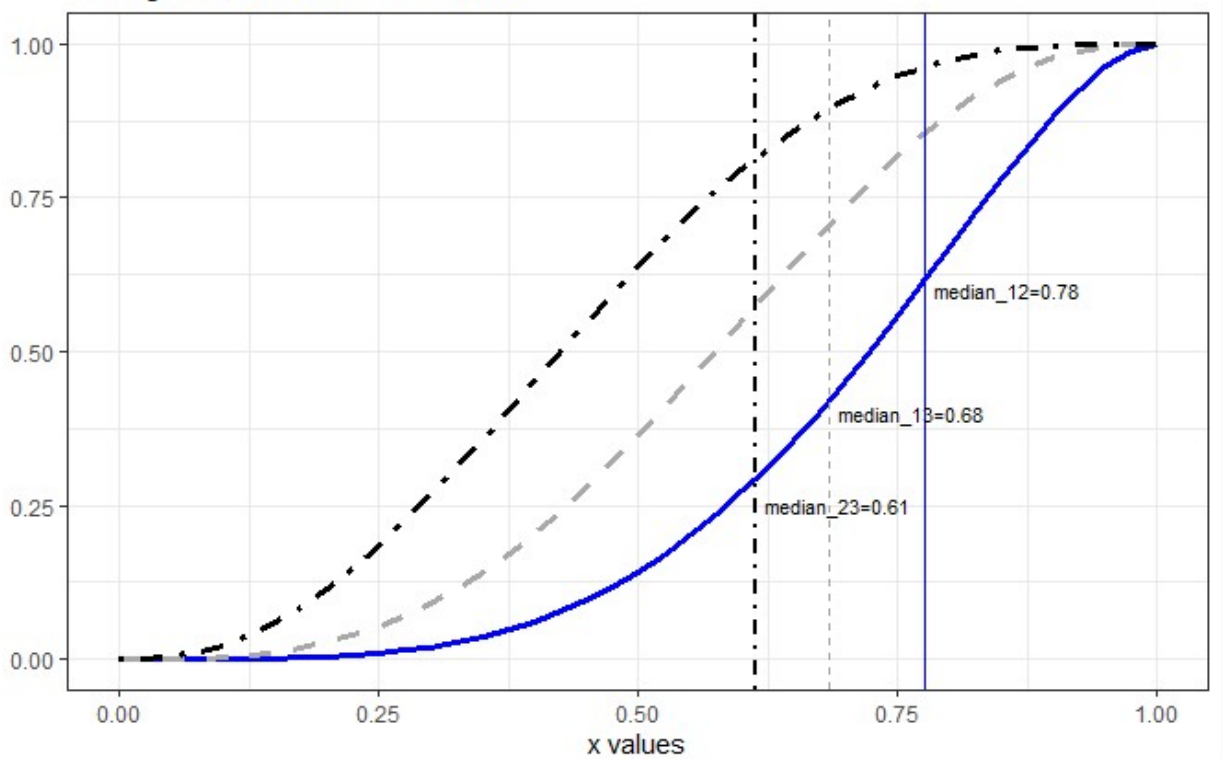

Fig. 7. Example of the asymmetrical triangular distribution functions (for stochastic coalition utilities $\tilde{v}_{1,2}, \tilde{v}_{1,3}, \tilde{v}_{2,3}$

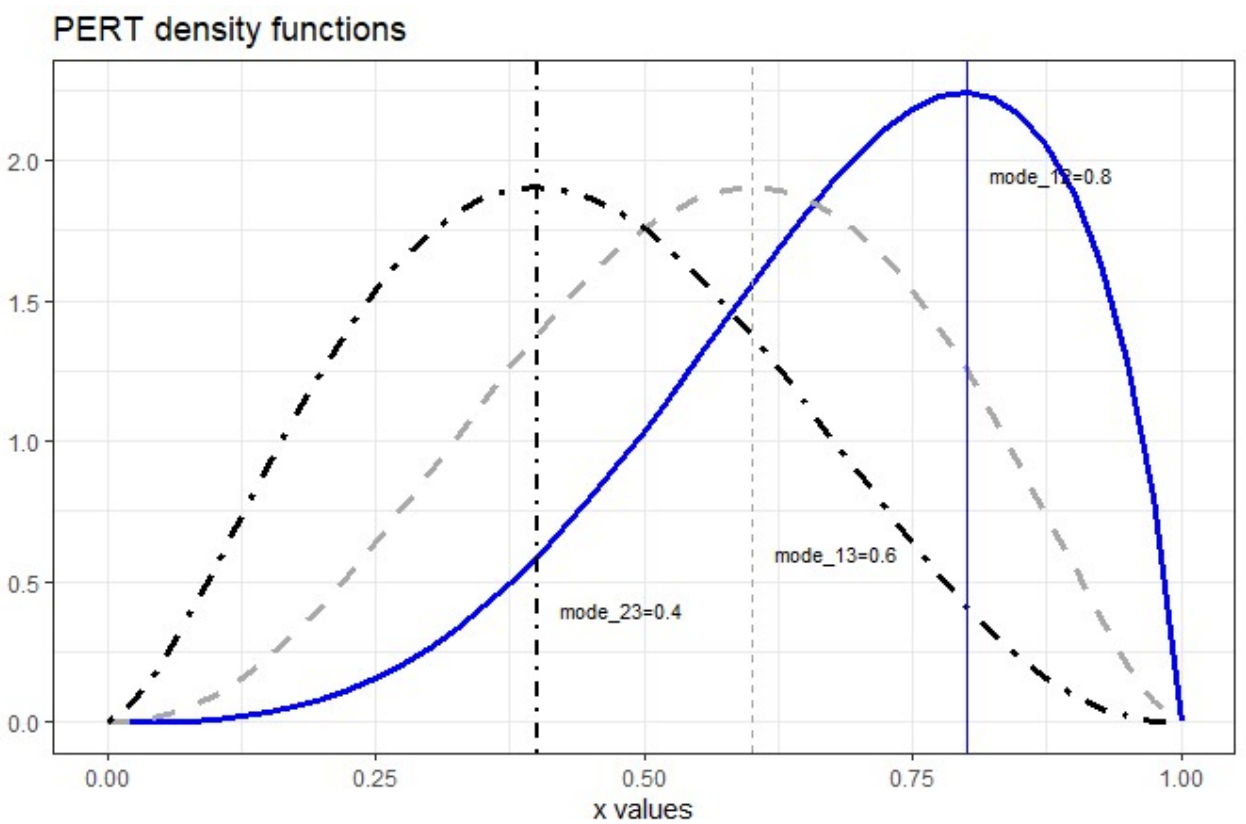

Fig. 8. Example of density for the PERT distribution functions (for stochastic coalition utilities $\tilde{v}_{1,2}, \tilde{v}_{1,3}, \tilde{v}_{2,3}$

\section{Conclusion}

The methods of the modern theory of cooperative games can act as effective tools for modeling and analyzing the processes of redistribution of political and economic-political influence between world centers of power. 


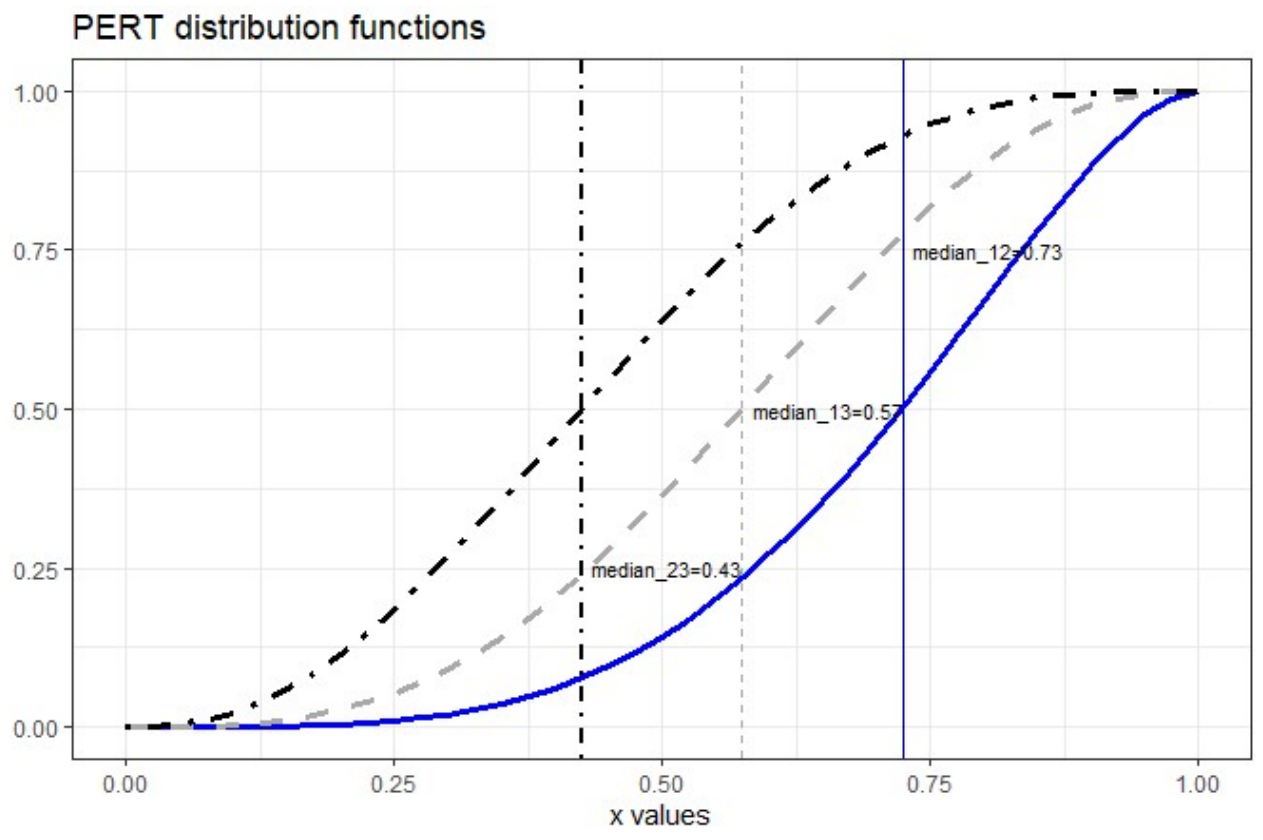

Fig. 9. Example of the PERT distribution functions (for stochastic coalition utilities $\tilde{v}_{1,2}, \tilde{v}_{1,3}, \tilde{v}_{2,3}$

Table 3. Modes of the distribution density functions of pairwise stochastic coalition utilities in a cooperative game with three participants (three centers of power)

\begin{tabular}{|l|l|l|l|}
\hline Version & $\tilde{v}_{1,2}$ & $\tilde{v}_{1,3}$ & $\tilde{v}_{2,3}$ \\
\hline I & 0.8 & 0.6 & 0.4 \\
\hline II & 0.9 & 0.5 & 0.2 \\
\hline
\end{tabular}

Source:conditional data

The examples given in this paper largely indicate that cooperative game models provide some internally consistent logic that explains the trends in accordance with which relationships have evolved and the redistribution of influence between world centers of power has taken place over the past decades.

A condition for the successful development of cooperative models of interaction of centers of power is, on the one hand, the improvement of methods for constructing characteristic functions in the direction of increasing the level of adequacy of taking into account the objective interests (utility) of countries and coalitions.

On the other hand, the work on improving the level of the mathematical apparatus used in the models, and, in particular, the apparatus of stochastic cooperative games, is very important in terms of the development of possible solution concepts, on the basis of which conclusions are drawn regarding the parameters of possible inter-coalition agreements. 
Table 4. Values of distributions $x_{1}, x_{2}, x_{3}$, forming a stochastic bargaining set in a game with three participants

\begin{tabular}{|l|l|l|l|l|l|l|}
\hline & \multicolumn{3}{|c|}{ Version I } & \multicolumn{3}{c|}{ Version II } \\
\hline$\alpha$ & $x_{1}$ & $x_{2}$ & $x_{3}$ & $x_{1}$ & $x_{2}$ & $x_{3}$ \\
\hline 0.95 & 0.27 & 0.13 & -0.03 & 0.37 & 0.13 & -0.15 \\
\hline 0.90 & 0.30 & 0.17 & 0.02 & 0.39 & 0.16 & -0.11 \\
\hline 0.85 & 0.31 & 0.18 & 0.04 & 0.40 & 0.18 & -0.08 \\
\hline 0.80 & 0.33 & 0.20 & 0.06 & 0.42 & 0.20 & -0.06 \\
\hline 0.75 & 0.35 & 0.22 & 0.08 & 0.43 & 0.21 & -0.04 \\
\hline 0.7 & 0.36 & 0.24 & 0.10 & 0.44 & 0.23 & -0.03 \\
\hline
\end{tabular}

Source:conditional data

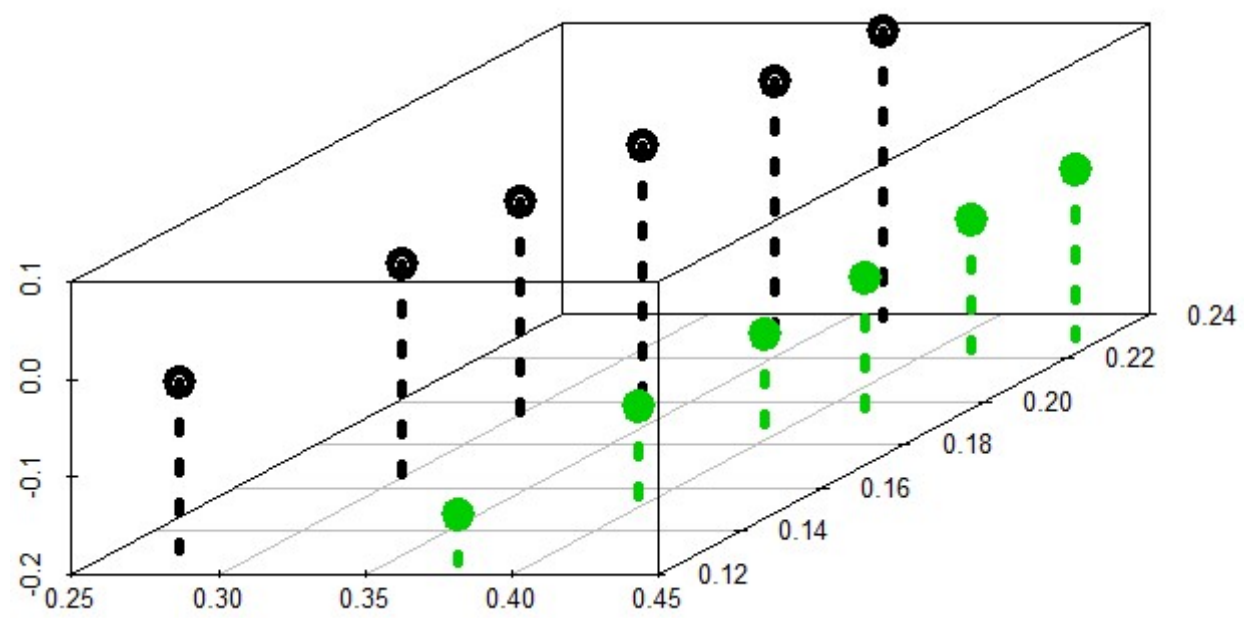

Fig. 10. Series of vector values depending on the probability levels $\alpha$ for versions I and II

\section{References}

Aleskerov, F. T., Kravchenko, A.S. (2008). The distribution of influence in the government of the Russian Empire Dumas.. Politeia, 3(50).

Aumann, R.J., Maschler, M. (1961). The Bargaining Set for Cooperative Games. Econometric Research Program Research Memorandum No. 34, 6 November 1961.

Banzhaf, J. F. (1965). Weighted voting doesnв $\hbar^{\text {TM }}$ t work: a mathematical analysis. Rutgers Law Review., 19, 317-343.

Blagden, D. (2019). Power, polarity, and prudence: the ambiguities and implications of UK discourse on a multipolar international system. Defence Studies. Published online. DOI: $10.1080 / 14702436.2019 .1643243$,

Charnes, A. and D. Granot (1977). Coalitional and Chance-Constrained Solutions to $n$ Person Games, II: Two-Stage Solutions. Operation Research 25(6), 1013-1019.

Charnes, A., Granot, D. (1973). Prior solutions: extensions of convex nucleolus solutions to chance-constrained games. In: Proceedings of the Computer Science and Statistics Seventh Symposium at Iowa University. pp. 1013-1019.

Coleman, J.S. (1971). Control of Collectivities and the Power of a Collectivity to Act. In: B. Lieberman (ed.) Social Choice, New York: Gordon and Breach, pp. 269-300. 
Deegan, J., Packel, E. W. (1978). A New Index of Power for Simple n-Person Games. In: International Journal of Game Theory, 7(2).

Degterev, D. A. (2020). Assessment of the current balance of power in the international arena and the formation of a multipolar world.. M .: Ru-science. (https://ruscience.com/ru).

Holler, M. J., Packel, E. W. (1983). Power, Luck and the Right Index. In: Journal of Economics, 43.

Johnston, R. J. (1978). On the Measurement of Power: Some Reactions to Laver. In: Environment and Planning., 10.

Keersmaeker, G. (2015). Multipolar Myths and Unipolar Fantasies. In: Security Policy Brief No 60. Brussels, Egmont Royal Institute for International Relations.

Konyukhovskiy, P. V. (2012) The use of stochastic cooperative games in the justification of investment projects. In: Vestnik St.Petersburg University. Ser.5 Economy, 4, 134-143.

Konyukhovskiy, P. V., Holodkova, V. V. (2017). Application of Game Theory in the Analysis of Economic and Political Interaction at the International level. In: Contributions to Game Theory and Management., Vol X

Pechersky, S. L., Yanovskaya, E. B. (2004). Cooperative Games: solutions and axioms. SPb.: Publishing House of Europe University of St. Petersburg.

Penrose, L. S. (1946). The Elementary Statistics of Majority Voting. Journal of the Royal Statistical Society, 109, 53-57.

Rae, D. W. (1969). Decision-Rules and Individual Values in Constitutional Choice. American Political Science Review, 63, 40-63.

Raymind, G.V. (2018). Advocating the rules-based order in an era of multipolarity. Australian Journal of International Affairs. Published online. DOI: $10.1080 / 10357718.2018 .1520803$

Schmeidler, D. (1969). The nucleolus of a characteristic function game SIAM Journal of Applied Mathematics, 17(6), 1163-1170.

Shapley, L., Shubik, M.A. (1954). Method for Evaluating the Distribution of Power in a Committee System. American Political Science Review, 3 Vol. 48.

Sokolova, A. V. (2008). Quantitative methods of assessing the impact of participating in the collective decision-making $\mathbf{5 1}$ Politeia 4.

Suijs, J., Born, P. (1999). Stochastic Cooperative Games: Superadditivity, Convexity, and Certainty Equivalents Games and Economic Behavior, 27, 331-345.

Suijs J.P. M. (1999). A nucleolus for stochastic operative games In J.P.M. Suijs (Ed.), Cooperative Decision-Making Under Risk. 1999. Boston: Kluwer Academic Publishers, pp. 152-181.

Suijs, J. P. M., Borm, P.E. M., De Waegenaere, A. M. B., Tijs S. H. (1999). Cooperative games with stochastic payoffs European Journal of Operational Research, 113(1), 193205.

Trush, S. M. (2020). RUSSIA-USA-CHINA: Resons and Risks of the Russian-Chinese Military Relationship Bulletin of the Russian Academy of Sciences, 90(11), 1037-1047.

Yeung, D.W.K., Petrosyan, L.A. (2006). Cooperative Stochastic Differential Games Springer, 2006.

Yeung, D.W.K., Petrosyan, L. A. (2004). Subgame consistent cooperative solutions in stochastic differential games J.Optimiz. Theory and Appl., 120(3), 651-666. 